\title{
Structural basis of SARS-CoV-2 translational shutdown and programmed ribosomal frameshifting
}

\author{
Nenad Ban
}

ETH Zurich, Zurich, Switzerland

We are investigating bacterial and eukaryotic ribosomes and their functional complexes to obtain insights into the process of protein synthesis. Building on our studies aimed at revealing the structures of eukaryotic cytosolic and mitochondrial ribosomes, we are now investigating eukaryotic translation initiation, targeting of proteins to membranes, regulation of protein synthesis, and how viruses reprogram host translation. Previously, we studied how Hepatitis C virus genomic RNA can bind mammalian ribosomes to achieve translation of viral mRNAs in the absence of some canonical cellular translation initiation factors. With our recent research activities we contributed to the understanding of how SARS-CoV-2, the virus that is responsible for the COVID-19 pandemic, shuts off host translation to prevent cellular defence mechanisms against the virus (Schubert et al. 2020). Furthermore, using a combination of cryoelectron microsocpy and biochemical assays we also investigated the mechanism of programmed ribosomal frameshifting, one of the key events during translation of the SARS-CoV-2 RNA genome that leads to synthesis of the viral RNA-dependent RNA polymerase and downstream viral proteins (Bhat et al. 2021).

Schubert K, Karousis ED, Jomaa A, Scaiola A, Echeverria B, Gurzeler LA, Leibundgut M, Thiel V, Mühlemann O, Ban N. (2020) SARS-CoV-2 Nsp1 binds the ribosomal mRNA channel to inhibit translation. Nat Struct Mol Biol. (10):959-966

Bhatt PR, Scaiola A, Loughran G, Leibundgut M, Kratzel A, McMillan A, O’ Connor KM, Bode JW, Thiel V, Atkins JF and Ban N, 2021, Structural basis of ribosomal frameshifting during translation of the SARS-CoV-2 RNA genome, Science, doi: 10.1126/science.abf3546.

Keywords: cryo-electron microscopy, protein synthesis, viral translation 